\title{
MIPYMES DISTRIBUIDORAS TURÍSTICAS: RECOMENDACIONES SEO A PARTIR DEL ANÁLISIS DE PALABRAS CLAVES
}

\author{
FRANCISCO EMANUEL GARCÍA GONZÁLEZ1 \\ Investigador Universidad Politécnica Salesiana - Grupo Investigación Gestión de las \\ MIPYMES - GIGMP \\ JUAN PABLO VÁZQUEZ LOAIZA ${ }^{2}$ \\ Investigador Universidad Politécnica Salesiana - Grupo Investigación Gestión de las \\ MIPYMES - GIGMP
}

\begin{abstract}
RESÚMEN
En las prácticas del comercio electrónico, las acciones desempeñadas a través del reconocimiento de palabras claves contribuyen una ventaja competitiva para las empresas, dado que la costumbre del turista por buscar información y gestionar sus viajes, provoca para las marcas, la necesidad de mostrarse y visibilizarse a través de las plataformas web. En dicho sentido, con el propósito de reconocer la realidad en cuanto a la explotación de las palabras claves en los portales web de empresas distribuidoras turísticas cuencanas, la investigación aplicó procedimientos de exploración y análisis cuantitativos y cualitativos desarrollados tanto en SPSS como en Nvivo. Estudios que permitieron descubrir que la gestión de palabras claves en las agencias de viaje, parecería aún no ser una prioridad, o que termina por ser ineficiente. Hallazgo que ha contribuido a deducir que la recomendación básica, con la cual se pueda aportar al contexto social, es la influencia sobre la importancia del uso de palabras claves y su impacto, tanto en la promoción de destinos turísticos, como en la generación de valor orientada a la personalización o individualización de productos.
\end{abstract}

PALABRAS CLAVES: distribución turística, posicionamiento web, gestión de valor

ABSTRACT: In the practices of electronic commerce, the actions carried out through the recognition of keywords contribute a competitive advantage for companies, given that the custom of tourists to seek information and manage their trips, causes for brands, the need to show and visualize through web platforms. In this sense, with the purpose of recognizing the reality regarding the exploitation of the keywords in the web portals of tourism distributing companies in Cuenca, the research applied quantitative and qualitative exploration and analysis procedures developed in both SPSS and Nvivo. Studies that allowed us to discover that the management of keywords in travel agencies, would seem not yet to be a priority, or that ends up being inefficient. Finding that has helped to deduce that the basic recommendation, with which it can contribute to the social context, is the influence on the importance of the use of keywords and their impact, both in the promotion of tourist destinations, and in the generation of value oriented to the customization or individualization of products.

KEYWORDS: Keywords, tourism distribution, web positioning, value management

\footnotetext{
1 fgarciag@est.ups.edu.ec

2 jvazquez@ups.edu.ec
} 


\section{INTRODUCCIÓN}

En el ámbito de las prácticas asociadas al comercio digital, la optimización de motores de búsqueda (SEO) es reconocida como la colección de técnicas (Silva, y otros, 2014) coadyuvantes a mejorar la visibilidad de un sitio web en los buscadores de Internet. De tal manera que, en beneficio para las marcas y establecimientos electrónicos, se pretenda alcanzar un posicionamiento alto en los resultados de búsquedas orgánicas. Entendido esto, en el hecho de que el nombre comercial se visibilice en las primeras páginas de los mismos (Rasekh, 2015) y cuyo resultado se deriva de la exploración al contenido web a partir de la indexación de palabras claves específicas dirigidas (Lukito, y otros, 2015).

En este contexto, la investigación se apega a la conceptualización aportada por Yalçn, y otros (2010) y Taylor (2011), en cuanto a reconocer a las palabras claves como aquellas expresiones que, para el usuario de un navegador de Internet, representan conceptos significativos y que están asociados al propósito de búsqueda (Silva, y otros, 2014). Términos que además provocan que, en la página de resultados del buscador, se devuelvan los enlaces a sitios web que guardarían correspondencia a las mismas. Prácticas que vistas desde la perspectiva empresarial, se convierten en una necesidad técnica que favorece a visibilizar las marcas y por ende a mejorar la captura de clientes, convirtiéndose en una experiencia de publicidad competitiva (Qiao, y otros, 2017).

Así, aunque la optimización puede llevarse a cabo también a través de la configuración de un diseño atractivo del sitio web (Grzywaczewski, y otros, 2010), el uso de palabras claves, que también fuera reconocido por el mismo autor, representa la alternativa idónea dado que ayuda a conjugar conceptos propios de la marca, como aquellos otros similares usados por los competidores y por su puesto por los clientes. Por lo tanto, para las empresas que desarrollan actividades de información y comercio a través de medios electrónicos, es necesario reconocer aquellos términos exactos que usan los internautas, propósito que se consigue en primer lugar con su identificación y posteriormente con la determinación del número de veces que cada término aparece en el resultado de búsqueda — número de impresiones - y la cantidad consumida por el usuario - posición media - (Chandran, y otros, 2016).

Por otro lado, en el contexto del turismo, la injerencia de las Tecnologías de la Información y Comunicación (TIC) no solo ha transformado la cultura de negocio de las empresas distribuidoras (Garbin, 2006) sino, además, se ha estimulado la aparición de un nuevo tipo de turista que, gracias a las bondades del Internet, ha logrado acceder a mayor variedad de productos, precios e información. Convirtiéndose en un perseverante usuario que busca paquetes o alternativas turísticas, obligando a que las agencias de viajes emprendan estrategias de promoción, información y mantenimiento de sus portales a fin de pretender una personalización y adaptación cultural del cliente, permitiendo la construcción y posicionamiento de una marca (Rovira, y otros, 2010).

Hecho que ha convertido a los sitios web en el primer punto de contacto para la introducción y promoción de los servicios (Maguire, y otros, 2016), la reserva de transporte y alojamiento (Ling, y otros, 2014). Por lo cual, al ser el Internet la fuente predominante para planificación de viajes, la calidad de la presencia en línea de una marca (Alcántara, y otros, 2017) se ha constituido en un factor crítico para la comunicación y la gestión de las relaciones con los clientes (Ye, y otros, 2017).

Así entonces, emprender una estrategia de mercadeo electrónico para los distribuidores turísticos, demanda un sinnúmero de acciones, de entre las cuales el SEO, es 
una de ellas. Y está centrado en visibilizar el dominio empresarial a través de los motores de búsqueda, ya que de nada sirve construir un sitio web atractivo y eficaz desde el punto de vista de la comunicación y la comercialización del destino turístico, si después, cuando el potencial turista realiza su búsqueda en la Red, es incapaz de encontrarlo o, termina navegando por una web no oficial (Egri, y otros, 2014).

Además, porque desde una perspectiva de segmentación de mercado, la determinación de palabras claves puede resultar una ventaja competitiva para las pequeñas y medianas empresas (PYMES), en la medida que les permite dirigirse a sectores específicos de turistas, como locales, nacionales o internacionales, o también, especializar sus productos a un tipo de turismo particular (p.ej., naturaleza, cultura, negocios). Por ello, sus sitios web deben ofrecer una ventaja distintiva (Tang, y otros, 2012) que provoca un efecto positivo hacia los destinos turísticos (Alcántara, y otros, 2017) y proporciona una experiencia al consumidor (Gao, y otros, 2014). De tal suerte que las prácticas efectivas contribuyan a la generación de valor, con lo cual, según Gómez, y otros (2015), a su vez se logre potenciar el desarrollo de los destinos turísticos

En este sentido, con el propósito de proporcionar información significativa para la gestión digital de distribución turística de la ciudad de Cuenca a fin de que se contribuya al mejoramiento de prácticas de comercio electrónico, la investigación se desarrolló considerando como antecedentes, los hallazgos detectados por el Grupo de Investigación de la Gestión de las Mipymes (GIGMP), en donde: a) en primer lugar, a través de un estudio preliminar aplicado a 38 agencias de viajes, se reconoció mediante un instrumento propuesto por Pérez, y otros (2012) que, la calidad de sus sitios web, es deficiente, y b) que asimismo, un estudio realizado por Vázquez, y otros (en prensa), demostró que las empresas de viajes cuencanas evidencian una insuficiente preparación en cuanto al aprovechamiento de las TIC para interactuar a través de páginas web con los turistas.

Por lo tanto, en el objetivo de contribuir a la generación de valor a través de la práctica de tecnologías digitales, la investigación se constituye como un insumo complementario que ayuda a evidenciar el comportamiento de las agencias de viajes cuencanas, a fin de promover la delineación de proyectos. Para lo cual, en torno a la orientación de las palabras claves que deberían estar configuradas en los sitios web de los distribuidores turísticos, se reconoce como preámbulo que el comportamiento del turista que llega a Cuenca, según Estrella y otros (2016) gira sobre los ámbitos de naturaleza, cultura y aventura, además que no se reconocen mercados ni productos especializados

Así entonces, el trabajo realizado a través de los enfoques cualitativos y cuantitativos logró establecer recomendaciones para la gestión digital de los sitios web de las agencias de viajes cuencanas, luego de estudiar la hipótesis que se reconoció en relación a saber si dichas distribuidoras turísticas estarían configurando adecuadamente las palabras claves. De tal manera que exista una orientación hacia un tipo de agencia o turismo en particular, además a la promoción del destino turístico Cuenca.

\section{METODOLOGÍA}

Para el desarrollo de la investigación en primer lugar, se determinó el objeto de estudio. Para el efecto, en base a un total de 144 empresas inventariadas en el Ministerio de Turismo del Ecuador, mediante una exploración realizada a través de navegadores de Internet, se detectó que apenas un número de 35 agencias de viajes de la ciudad de Cuenca han contratado un dominio web, por lo cual el estudio no se llevó a cabo a través de muestreo sino mediante un censo que involucró al total de dichas empresas. 
Posterior a ello, con el propósito de identificar las palabras claves configuradas en los sitios web de las agencias de viajes, se utilizó la herramienta Metricspot, misma que fue seleccionada en consideración de: 1) ser una aplicación de acceso gratuito, 2) la facilidad de uso según recomendación de Archanco (2017), 3) la incorporación de cerca de 80 indicadores para análisis de posicionamiento web agrupados las categorías Autoridad SEO, SEO básico, Contenido, Usabilidad, Aspectos Técnicos y Redes Sociales, 4) no limita el uso de páginas web para el análisis (Lleida, 2016), y porque 5) no es un planificador de palabras claves sino una herramienta analítica.

Una vez reconocidas las palabras claves, el análisis de los datos se procedió a través de herramienta SPSS versión 23, en la cual se realizó el análisis estadístico descriptivo mediante el análisis de frecuencias, a fin de determinar las palabras con mayor aparición entre las empresas, resultados que fueron analizados mediante representación de Diagramas de Venn y lógica de conjuntos.

El estudio se complementó a través del Índice de Similitud de Jaccard (IJ) mostrado en la ecuación 1, y que se aplicó para confirmar el grado de similitud de palabras claves entre las agencias de viajes. Para lo cual, en conformidad a lo explicado por Villégarab y otros (2012), la investigación consideró el valor de 1 para representar existencia de similitud y 0 para el caso contrario.

$$
I_{j}=\frac{a}{a+b}
$$

En donde:

$I_{j}=$ se reconoce como Índice de Jaccard

$\mathrm{a}=$ corresponde al número de palabras claves comunes

$\mathrm{b}=$ representa el número de palabras claves no comunes

Desde el ámbito cualitativo, en apoyo a la herramienta Nvivo versión 11, con el propósito de profundizar el estudio de correspondencia de las palabras claves, dado que posterior a la aplicación del IJ, estadísticamente no se determinó correspondencia significativa. Se sistematizaron las palabras con relación a tres nodos de análisis que buscaron establecer similitud alguna en función de: a) Tipo de Agencia, en donde se distinguió a las empresas Duales, Internacionales, Mayoristas y Operadoras, b) Destino Turístico para reconocer la orientación en cuanto a turismo emisor o receptor y que distinguiera a promociones de tipo Regional, Nacional e Internacional, y c) Tipo de Turismo, a través del cual se distingue el propósito del turismo (p.ej. montaña, cultura).

Dicha correspondencia se validó a través de tablas cruzadas, en donde a partir de consultas configuradas también en Nvivo, se descubrió la relación en dos sentidos: 1) Tipo de Agencia versus Destino turístico, para determinar el horizonte de cumplimiento comercial de las empresas con relación al producto turístico que ofertan, y 2) Tipo de Agencia vs Tipo de Turismo, para buscar una especialización en la gestión publicitaria de los productos a los cuales se orientan cada uno.

\section{RESULTADOS}

Tras la aplicación metodológica, la presente sección presenta los resultados más relevantes de la investigación. A la par que describe, analiza y discute la realidad de las empresas distribuidoras turísticas estudiadas. 


\subsection{Exploración de palabras claves}

Por intermedio del uso de la herramienta Metricspot, el primer paso del estudio determinó un total de 410 términos diferentes extraídos desde la nube de palabras claves configuradas dentro del código fuente los sitios web. Cuyo conjunto se ejemplifica en la figura 1, en donde se aprecia la colección de palabras y su número de impresiones. Además, que evidenció que existen expresiones que en efecto hablan y se relacionan a la actividad turística, pero otros que parecerían ser irrelevantes para el efecto.



Figura 1. Ejemplo nube de palabras claves

Fuente: Elaboración propia del autor, extracción a través de Metricspot

Nota: Las cantidades mostradas en azul representan el número de impresiones de cada palabra clave

Desde el reconocimiento de las palabras claves, las tabas 1 y 2, representan en su orden: a) las 10 palabras más usadas en las empresas conforme la frecuencia estadística y reconocidas como conjunto (A), y b) las 10 más importantes conforme el número de impresiones y que se determinó a través de Metricspot, denominado para el estudio como conjunto (B). Sobre dicha exploración, se evidenció que el número de palabras claves según el análisis de frecuencias, dista de aquellas configuradas como importantes según el número de impresiones. Por lo tanto, parecería no existir una coherencia, entre lo que las empresas configuran entre sí, y la importancia que las mismas otorgan a los términos claves.

\begin{tabular}{|c|c|}
\hline Palabra & Frecuencia (fi) \\
\hline Cuenca & 8 \\
\hline días & 5 \\
\hline Ecuador & 17 \\
\hline Galápagos & 5 \\
\hline información & 5 \\
\hline tour & 8 \\
\hline tours & 12 \\
\hline travel & 5 \\
\hline ver & 5 \\
\hline viajes & 6 \\
\hline
\end{tabular}

Tabla 1. Palabras más importantes conforme frecuencia entre empresas

Fuente: Elaboración propia del autor a través de SPSS 


\begin{tabular}{|c|c|}
\hline Palabra & $\mathbf{N}^{\circ}$ impresiones \\
\hline ahorra & 25 \\
\hline click & 33 \\
\hline day & 28 \\
\hline Ecuador & 38 \\
\hline opens & 33 \\
\hline precio & 26 \\
\hline share & 34 \\
\hline trip & 25 \\
\hline ver & 25 \\
\hline window & 33 \\
\hline
\end{tabular}

Tabla 2. Palabras más importantes conforme impresiones configuradas en web site

Fuente: Elaboración propia del autor a través de Metricspot

Así, con relación a dichos conjuntos, con el propósito de determinar las palabras más importantes entre los mismos, se aplicó la intersección $(\mathrm{A} \cap \mathrm{B})$, representada en el Diagrama de Venn de la figura 2, y que reconoció a: 1) la palabra Ecuador como la más relevante y que denota el interés por prácticas de turismo receptor, y 2) la palabra ver, que podría considerarse irrelevante puesto que no estaría asociada al desarrollo de las actividades turística pero que, sin embargo, son comunes en la configuración dentro de las páginas web turísticas.



Figura 2: Intersección conjuntos $(\mathrm{A} \cap \mathrm{B})$, palabras claves más importantes Fuente: Elaboración propia del autor

Asímismo, con el propósito de aportar un mayor análisis a los datos, a través de la figura 3, se configuraron consultas para establecer el conjunto de palabras que evidencien: a) aquellas más relevantes en relación a la actividad turística (figura 3a), b) los términos aparentemente irrelevantes y que podrían representarse por aquellas que no corresponderían a un propósito adecuado conforme la actividad turística (figura 3b), y c) las expresiones asociadas a los destinos, a fin de descubrir cuales son los lugares sobre los cuales las agencias centrarían su prioridad (figur 3c). 




(a) Palabras relevantes

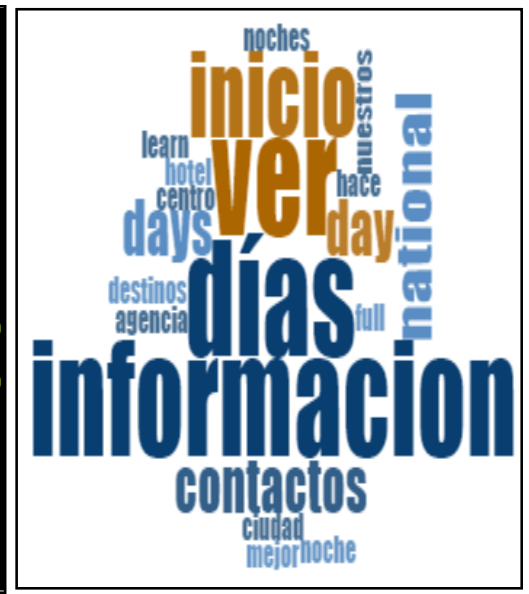

(b) Palabras irrelevantes

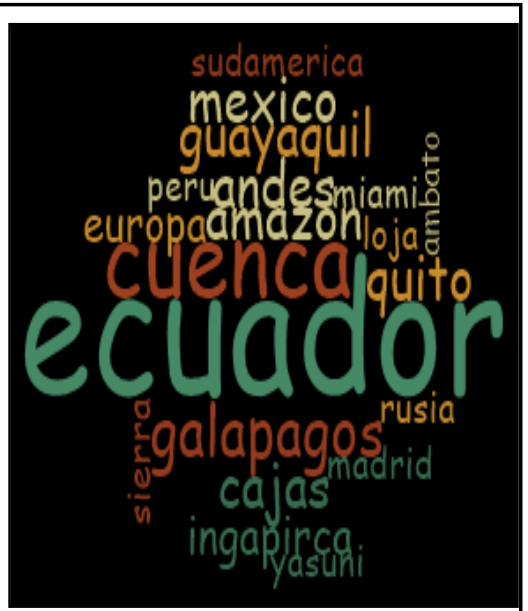

(c) Destinos turísticos

Fuente: Elaboracion propia del autor, extracción a través de Nvivo

Nota: Compuesta por las 20 palabras claves de mayor frecuencia.

\subsection{Estudio de similitud}

El estudio de la similitud de palabras claves calculado con IJ devolvió un p-valor de similitud que oscila entre 0,13 y 0,250. Con lo cual, al no encontrarse valores estadísticamente significantes cercanos a 1 , se determina una ausencia de similitud entre las empresas y se rechaza la dicha hipótesis, entendiéndose que entre las empresas no existen concordancia en cuanto a conceptos turísticos con orientación al marketing.

Dicho resultado fue corroborado con el análisis en la herramienta Nvivo, desde el cual se construyó el dendrograma vertical (figura 4) a partir del IJ, ante lo cual, se obtuvo una alta dispersión de palabras entre las empresas agencias de viajes. De esta manera, el IJ indica que existen empresas distribuidoras con cero similitudes, lo cual podría entenderse en dos horizontes: 1) que las empresas no estarían comprometidas en realizar dicha configuración técnica en sus archivos web, o 2) que existiría una especialización en cuanto a las preferencias de cada empresa.



Figura 3. Agrupación de agencias de viajes según similitud de palabras claves

Fuente: Elaboración propia del autor, extracción a través de Nvivo 


\section{a. Análisis de correspondencia}

La consecución del objetivo se logró además desde un enfoque cualitativo apoyado en Nvivo y que, a través de la inspección de palabras claves bajo un razonamiento lógico, se logró identificar la correspondencia de cada una de ellas en cuanto a nodos de análisis. En donde en la figura 4, se representan los conjuntos conforme al nodo tipo de agencia, reconociendo la prioridad de términos que las agencias Duales (C), Internacionales (D), Mayoristas (E) y Operadoras (F) han configurado, y cuya intersección $(C \cap D \cap E \cap F)$ se representa en la figura 5 y cuyo resultado coincide con la palabra Ecuador y que se representa ya anteriormente.



Figura 4. Conjunto de palabras claves conforme el nodo tipo de agencia Fuente: Elaboración propia del autor




Figura 5: Intersección conjuntos $(\mathrm{C} \cap \mathrm{D} \cap \mathrm{E} \cap \mathrm{F})$ por tipo de agencia Fuente: Elaboración propia del autor

Posteriormente, profundizando el análisis, se configuró una tabla cruzada entre los nodos Tipo de agencia y Destino turístico (tabla 3). Aquí se observa la frecuencia de palabras para cada combinación que se están utilizando actualmente por las empresas en sus respectivas páginas web. Se identifican al respecto con mayor presencia la combinación agencia Operadora vs destino Nacional encontrando palabras como (Cuenca, Ecuador, Galápagos, Quito, Guayaquil), lo cual indica que se está utilizando coherentemente las palabras claves, es decir se encuentran bien orientadas a su mercado.

En cuanto a empresas Duales se conoció, que están orientadas en mayor nivel igualmente a un turismo dentro del territorio nacional promocionando destinos principalmente como (Galápagos, Grayline, Amazon, Quito, Guayaquil, Yasuní). Por su parte empresas Internacionales contra destinos Internacionales, tiene una correcta identificación de palabras claves al igual que su orientación al segmento correspondiente, finalmente seguidos por las Mayoristas que no se encuentran adecuadamente orientadas a ninguno de los destinos propuestos.

\begin{tabular}{l|ccc}
\hline \multicolumn{4}{c}{ Destinos Turísticos } \\
\hline $\begin{array}{l}\text { Tipo } \\
\text { Agencia }\end{array}$ & Internacional & Nacional & Regional \\
\hline Duales & 13 & $\mathbf{2 0}$ & 3 \\
Internacionales & $\mathbf{2 0}$ & 5 & 1 \\
Mayoristas & 4 & 2 & 1 \\
Operadoras & 8 & $\mathbf{2 2}$ & 15 \\
\hline
\end{tabular}

Tabla 3. Nodos tipo de agencia versus destinos turísticos

Fuente: Elaboración propia del autor

Nota: Con negrita se representa la relación con mayor frecuencia.

Seguido, se analizó la relación que integran los nodos tipo de agencia versus el tipo de turismo. De lo cual, a través de la tabla 4, se representa el número de palabras que corresponden a la intersección de los nodos, dando a conocer así que, para las distribuidoras turísticas Duales e Internacionales el mayor turismo se da gracias a experiencias de Aventura donde se integran por ejemplo kanyoning, alpinismo y ciclismo, seguido de cerca con las experiencias de Montaña a través de representaciones como birding, hiking, paisajes y forest, actividades que dicho sea de paso, podrían referir a destinos cercanos o fuera de Cuenca. En cuanto a las Operadoras, existió gran diferencia entre ambos tipos de turismo. Finalmente, para empresas Mayoristas se identificó que no se encuentra orientada a ningún tipo de turismo (p.e., educativo, medicinal, congresos, cultural), ya que las mismas no pueden ofrecerlos directamente al consumidor.

\begin{tabular}{l|ll}
\hline & \multicolumn{2}{l}{ Tipo de Turismo } \\
\hline $\begin{array}{l}\text { Tipo de } \\
\text { Agencia }\end{array}$ & Aventura & Montaña \\
\hline Duales & 13 & 10 \\
Internacionales & 6 & 3 \\
Mayoristas & 0 & 0 \\
Operadoras & $\mathbf{3 1}$ & $\mathbf{2 1}$ \\
\hline
\end{tabular}


Tabla 4. Nodos tipo de agencia versus tipo de turismo

Fuente: Elaboración propia del autor

\section{CONCLUSIONES}

En cuanto a la identificación de las palabras claves usadas por las empresas distribuidoras turísticas de la ciudad de Cuenca en sus páginas webs oficiales, se encontraron expresiones orientadas al sector turístico, pero en un mayor grado se logró identificar aquellos considerados como irrelevantes. Argumentos a través de los cuales se concluye que, entre las agencias de viajes, la práctica por las estrategias de identificación y uso de las mismas es aún insuficiente, puesto que ni siquiera otorgan importancia hacia el nombre comercial, dado que, a través del número de impresiones, ninguna resalta como relevante.

Al determinar la asociación de las palabras claves utilizadas en torno a los diferentes segmentos. Se han detectado también algunas falencias sobre el posicionamiento de los sitios webs oficiales de empresas distribuidoras turísticas de la ciudad de Cuenca, ya que en cuanto a la correspondencia entre los nodos tipo de agencia y tipo de destino, únicamente las agencias Internacionales usan palabras claves que corresponden a su mercado (Internacional). Mientras que, por su parte para empresas Duales, Mayoristas y Operadoras, no se encuentran orientadas a sus debidos segmentos o en algunos casos carecen de palabras clave relevantes dentro de la configuración de sus páginas.

En cuanto a la correspondencia con el tipo de turismo, se encontró evidencia que, tanto empresas Duales, Internacionales y Operadores, se orientan en mayor número a turismo de aventura. Mientras que por su parte las agencias Mayoristas carecen de alguna orientación, lo cual es satisfactorio ya que estas no pueden estar orientadas a ningún tipo de turismo sino únicamente a promocionar los destinos como tal. Al igual que lo que ofertan las Mayoristas, no necesariamente es replicado por las Duales e Internacionales.

Desde la perspectiva comercial, las empresas distribuidoras turísticas no están utilizando las palabras claves adecuadas dentro de la configuración de sus páginas webs, lo cual estaría digitalmente afectando sus actividades puesto que: 1) no estarían apareciendo entre los primeros resultados de los diferentes motores de búsqueda, lo que conlleva a un posicionamiento bajo en la mente de los internautas, 2) no estarían orientadas hacia un segmento de mercado específico y que se da por la falta de conocimiento de los diferentes mercados que existen. Lo cual confirmaría el hallazgo observado por Vázquez, y Gómez (en prensa) que, en efecto desde la orientación del modelo de negocios empresa-a-consumidor, las agencias de viajes cuencanas no estarían preparadas para contribuir a la gestión de valor en aprovechamiento de las TIC.

Finalmente, el análisis aquí realizado evidencia los resultados vistos desde el lado de las agencias de viaje. Sin embargo, con la finalidad de reconocer si esta realidad guarda concordancia con la perspectiva nacida del turista, se sugiere que el estudio sea complementado con el reconocimiento de palabras claves que los usuarios viajeros practican al momento de buscar información y gestionar el turismo. De tal forma que, únicamente con el diagnóstico total enfrentado desde ambas perspectivas, se podrá determinar si el enfoque comercial evidenciado en las plataformas web está adecuadamente orientado y poderse establecer las recomendaciones y estrategias necesarias para el caso. 


\section{BIBLIOGRAFÍA}

Alcántara, J. M., y otros. 2017. The antecedent role of online satisfaction, perceived risk online, and perceived website usability on the affect towards travel destinations. Journal of Destination Marketing \& Management. 2017.

Archanco, R. 2017. Papelesdeinteligencia. [En línea] 31 de Junio de 2017. http://papelesdeinteligencia.com/metricspot-herramienta-de-analisis-web/.

Chandran, M. y Ramani, A.V. 2016. Website Quality Evaluation Based on Search Engine Queries using Web Rank Position Algorithm (WRPA). 2016. págs. 224-230.

Egri, G. y Bayrak, C. 2014. The Role of Search Engine Optimization on Keeping the User on the Site. 2014. págs. 335-342.

Estrella, M, y otros. 2016. Plan Estratégico de Desarrollo Turístico y Mercadeo del destino Cuenca y su área de influencia 2016 - 2021. Plan estratégico de turisno de Cuenca. Cuenca : s.n., 2016. págs. 1140.

Gao, L. y Bai, X. 2014. Online consumer behaviour and its relationship to website atmospheric induced flow: Insights into online travel agencies in China. 2014. Vol. 21, págs. 653-665.

Garbin, D. 2006. Application of information and communication technologies (ICT) in tourism. International conference proceedings, Zarajevo, Bosnia y Herzegovina. 2006. págs. 925-932.

Gómez, G., Menoya, S. y Rodriguez, M. 2015. La cadena de valor como hilo conductor de la investigación en los destinos turísticos. Avances. 2015. Vol. 17, págs. 239-250.

Grzywaczewski, A., y otros. 2010. E-Marketing Strategy for Businesses. 2010. págs. 428-434.

Ling, L., Guo, X. y Yang, C. 2014. Opening the online marketplace: An examination of hotel pricing and travel agency on-line distribution of rooms. Tourism Management. 2014. Vol. 45, págs. 234-243.

Lukito, R. B., Lukito, C. y Arifin, D. 2015. Implementation techniques of search engine optimization in marketing strategies through the internet. 2015. págs. 1-6.

Lleida, T. 2016. Clicandwords. Herramientas SEO. 12 de Septiembre de 2016.

Maguire, Á., y otros. 2016. Raising concern about the information provided on medical travel agency websites: A place for policy. Health Policy and Technology. 2016. Vol. 5, págs. 414-422.

Pérez, R., Guzmán, M. y Pérez, M. 2012. Evaluación de la calidad de los sitios web que comercializan destinos turísticos cubanos. Estudios y perspectivas en turismo. 2012. Vol. 5, págs. 1281-1298.

Qiao, D., y otros. 2017. Finding competitive keywords from query logs to enhance search engine advertising. Information \& Management. 2017. Vol. 54, págs. 531-543.

Rasekh, I. 2015. A New Competitive Intelligence-Based Strategy for Web Page Search. 2015. págs. 450-456. 
Rovira, C., y otros. 2010. Posicionamiento en buscadores de las webs oficiales de capitales de provincia españolas. 2010. págs. 277-284.

Silva, N. y Aguiar, A. 2014. Web Site Optimization for Search Engines An Empirical Study. 2014. pág. Article number 6876888.

Tang, L., Jang, S. y Morrison, A. 2012. Dual-route communication of destination websites. Tourism Management. 2012. Vol. 33, págs. 38-49.

Taylor, D. 2011. Optimización del Search Engine de WordPress 3. Birmingham : Packt Publishing, 2011. págs. 79-82.

Vázquez, J. P. y Gómez, G. Las TIC como creación de valor al proceso de gestión de distribución turística para formulación de proyectos de adopción tecnológica. I D Tecnológico.

Villégerab, S. y Brosseab, S. 2012. Measuring changes in taxonomic dissimilarity following species introductions and extirpations. Ecological Indicators. 2012. Vol. 18, págs. 552-558.

Yalçn, N. y Köse, U. 2010. What is search engine optimization: SEO? 2010. págs. 487-493.

Ye, B. H., y otros. 2017. Website interactivity and brand development of online travel agencies in China: The moderating role of age. Journal of Business Research. 2017. 